\title{
CIN85 associates with endosomal membrane and binds phosphatidic acid
}

\author{
Jing Zhang ${ }^{1}$, Xiudan Zheng ${ }^{1}$, Xiao Yang ${ }^{1}$, Kan Liao ${ }^{1}$ \\ ${ }^{I}$ State Key Laboratory of Molecular Biology, Institute of Biochemistry and Cell Biology, Shanghai Institutes for Biological Sci- \\ ences, Chinese Academy of Sciences, Shanghai 200031, China
}

CIN85 (Cbl-interacting protein of $85 \mathrm{kDa}$ ) is an important molecule involved in receptor tyrosine kinase endocytosis. Here we report that through its positively charged C-terminus, CIN85 associates with a fusogenic lipid - phosphatidic acid. Its coiled-coil domain plays an important role in mediating this protein-lipid interaction. Deletion of the coiled-coil domain results in loss of membrane association, and reduced interaction with c-cbl, finally causing the blockage of epidermal growth factor receptor downregulation. In addition, a significant portion of CIN85 is located on the endosomal compartment and is related to endocytic cargo sorting, characterized by CIN85's localization on the "E class" compartment and EGF degradation blockage in CIN85 knockdown cells. Taken together, our results suggest that CIN85 may function as a scaffold molecule in both the internalization and endocytic cargo sorting processes through its association with the endosomal membrane.

Keywords: CIN85, the coiled-coil domain, phosphatidic acid, EGFR endocytosis, ESCRT assembly

Cell Research (2009) 19:733-746. doi: 10.1038/cr.2009.51; published online 5 May 2009

\section{Introduction}

CIN85 and the structurally related molecule CMS/ CD2AP belong to a family of ubiquitously expressed adaptor proteins that contain three $\mathrm{SH} 3$ domains, a proline-rich region and a coiled-coil domain [1]. CIN85 was first identified in human as a Cbl-interacting protein of $85 \mathrm{kDa}$ [2]. c-Cbl is a ubiquitin ligase that binds and ubiquitinates activated receptor tyrosine kinases (RTKs) during receptor internalization and downregulation [3]. It recruits CIN85 to the activated RTK to initiate the endocytotic process for receptor signaling inactivation [4, 5]. Once internalized, the receptor is sorted for recycling back to the cell surface or targeting to lysosomes for degradation. In the receptor degradation process, which is one of the main mechanisms for rapid clearance of activated RTKs from the cell surface [6], c-Cbl catalyzes multiubiquitination of the receptor as well as monoubiquitination of CIN85 [7]. This ubiquitinated receptor-CblCIN85 complex is then sorted into multivesicular bodies

\footnotetext{
Correspondence: Kan Liao

Tel: +86-21-54921113; Fax: +86-21-54921011

E-mail:kliao@sibs.ac.cn

Received 31 July 2008; revised 30 November 2008; accepted 31 December 2008; published online 5 May 2009
}

(MVB) that fuse into lysosomes $[8,9]$.

In the Cbl-CIN85-mediated receptor internalization process, the interaction between c-Cbl and CIN85 plays a crucial role in the initiation of receptor endocytosis. The interaction between CIN85 and c-Cbl is mediated by SH3 domains of CIN85 and the proline-rich motif (PXXXPR) in $\mathrm{c}-\mathrm{Cbl}[1,4,5,10]$. The proline-rich region in CIN85 itself is a binding site for many SH3 domain-containing proteins, such as endophilins, which are regulatory proteins that control endocytosis through clathrin-coated pits $[1,4,5,11,12]$. Through interaction with CIN85, RTK-Cbl is brought into proximity with endophilins and endocytotic pits. By interacting with c-Cbl through $\mathrm{SH} 3$ domains and with endophilins through the proline-rich region, CIN85 bridges RTK-Cbl and endocytotic proteins in an RTK-Cbl-CIN85-endophilin complex to mediate receptor endocytosis.

Besides the SH3 domains and the proline-rich region, there is a coiled-coil domain at the C-terminus of CIN85 [1]. Many proteins that are associated with endosomes and the cytoskeleton contain a similar type of coiled-coil domain [13]. In CMS/CD2AP, the coiled-coil domain is involved in the interaction with F-actin $[14,15]$. In CIN85, which has a much weaker F-actin-binding ability than CMS/CD2AP [15], the coiled-coil domain is required for CIN85 oligomerization and monoubiquitina- 
tion by $\mathrm{Cbl}$ [7]. The three $\mathrm{SH} 3$ domains or the prolinerich domain of CIN85 can cluster multiple effectors, while an individual $\mathrm{SH} 3$ domain has a relatively low affinity for the effectors. Hence CIN85 can rapidly exchange binding partners, depending on their local concentrations, cellular compartmentalization, and posttranslational modifications, in response to changes in the environment. These dynamic interactions explain why a high molecular-weight complex of $300-1000 \mathrm{kDa}$ is found in the native cell lysate, independent of stimulation [16]. However, the location of this complex within cells remains to be elucidated.

In this article, we report that CIN85 is associated with phosphatidic acid through its positively charged C-terminus. Its coiled-coil domain plays an important role in this interaction. Epidermal growth factor receptor (EGFR) downregulation and recruitment of Cbl by CIN85 are greatly reduced by deletion of the coiled-coil domain. In addition, CIN85 participates in the MVB sorting process by interacting with endosomal sorting complex required for transport (ESCRT) components. Furthermore, knockdown of CIN85 expression blocks EGF degradation. Therefore, our results suggest that CIN85 is an endosomal membrane-associated scaffold molecule that functions in both internalization and cargo-sorting processes.

\section{Results}

\section{CIN85 is located on cellular membranes}

It has been shown that CIN85 is present in a high molecular-weight complex under native conditions $[16$, 17]. However, the cellular localization of this complex remains unknown. It is reasonable to hypothesize that this complex is in some way connected to the intracellular membrane or cytoskeleton. In steady-state 3T3L1 cells that express high-level endogenous CIN85, we found that CIN85 was associated with the cellular membrane fractions, which have a similar density to Golgi vesicles ( $\gamma$-adaptin-marked membrane fractions) (Figure 1A). This localization is apparently different from those of many known molecular markers, such as N-cadherin (plasma membrane marker), Calnexin (ER marker) and $\gamma$-adaptin (Golgi marker).

Following differential centrifugation at $200000 \mathrm{~g}$, CIN85 was found to be in the pellet, which indicated that CIN85/Ruk is associated with the intracellular membrane. This pellet was insoluble in detergent, but could be dissolved in as little as $0.15 \mathrm{M} \mathrm{NaCl}$ or $25 \mathrm{mM} \mathrm{Na}_{2} \mathrm{CO}_{3}$ ( $\mathrm{pH}$ 11.0) (Figure 1B). These observations suggest that CIN85 is peripherally associated with the membrane, and might exist in a detergent-insoluble complex.

In immunofluorescence images, CIN85 is dispersive in the cytoplasm with small dot-like structures (Figure 1C). Over-expression of GFP- or HA-tagged CIN85 produced a more obvious punctate pattern (Figure 1D). It could be observed that CIN85 was located on the edges of some dark circular areas (see arrows in Figure 1D), part of which were filled with endocytic EGF (see arrows in Figures 1E, 4A, 5A and 5B). Some cells expressing exogenous CIN85 showed very large dot-like structures. However, these dots are not aggresomes, as they failed to co-localize with Hsp70 (a heat shock protein that acts as chaperone and is involved in the unfolded protein response) or $\gamma$-tubulin (an MTOC marker) (Supplementary information, Figure S1). These dots could be aggregates of CIN85 and its binding partners. Co-clustering of HA$\mathrm{c}-\mathrm{Cbl}$ and eGFP-CIN85 was directly observed in cells co-expressing HA-c-Cbl and eGFP-CIN85. Single c-Cbl is dispersively expressed in the cytoplasm. However, in all cells co-expressing GFP-CIN85 and HA-c-Cbl, it seems that $\mathrm{c}-\mathrm{Cbl}$ was recruited into the dot-like structures that were formed following CIN85 over-expression. Alix C-terminus was also found to co-localize with the dotlike structures (Supplementary information, Figure S1).

\section{CIN85 associates with phosphatidic acid via its posi- tively charged $C$-terminus}

The supercentrifugation results obtained from cells transfected with the truncated forms (Figure 2A) of CIN85 clearly show that CIN85 inner membrane localization required the whole PRC fragment. These data also show a correlation between the presence of the coiled-coil domain and membrane association (Figure $2 \mathrm{~B})$. The coiled-coil domain-deleted forms $(\triangle \mathrm{CC}, \mathrm{SH} 3$ and $\mathrm{PR}$ ) were more soluble than their counterparts with the coiled-coil domain (CIN85, SH3C and PRC) (Figure 2B).

No membrane-interacting modules have ever been reported in the CIN85 molecule, which is hydrophilic (PI 6.18 ) and lacks long hydrophobic amino-acid stretches. However, we found that the charge distribution of CIN85 was extremely biased. The $\mathrm{N}$-terminus containing three SH3 domains is acidic (PI 4.86), whereas the C-terminus PRC fragment is basic (PI 9.69). We wondered whether the asymmetrically charged CIN85 protein interacted with phospholipids through its basic region, as in the cases of mVps24 and hSnf7 [18, 19]. To address this question, we purified CIN85 fusion proteins and analyzed their ability to bind to a PIP strip membrane.

eGFP-CIN85 or eGFP- $\triangle$ CC expressed in HEK293 cells was purified and subjected to lipid-binding analysis (Supplementary information, Figure S2). Of 15 different phospholipids on the PIP Strips ${ }^{\mathrm{TM}}$ membrane, the purified eGFP-CIN85 fusion protein could interact only with 
A $1 \quad 2 \quad 3 \quad 4 \quad 5 \quad 6 \quad 7 \quad 8 \quad 9 \quad 10111213141516171819202122$
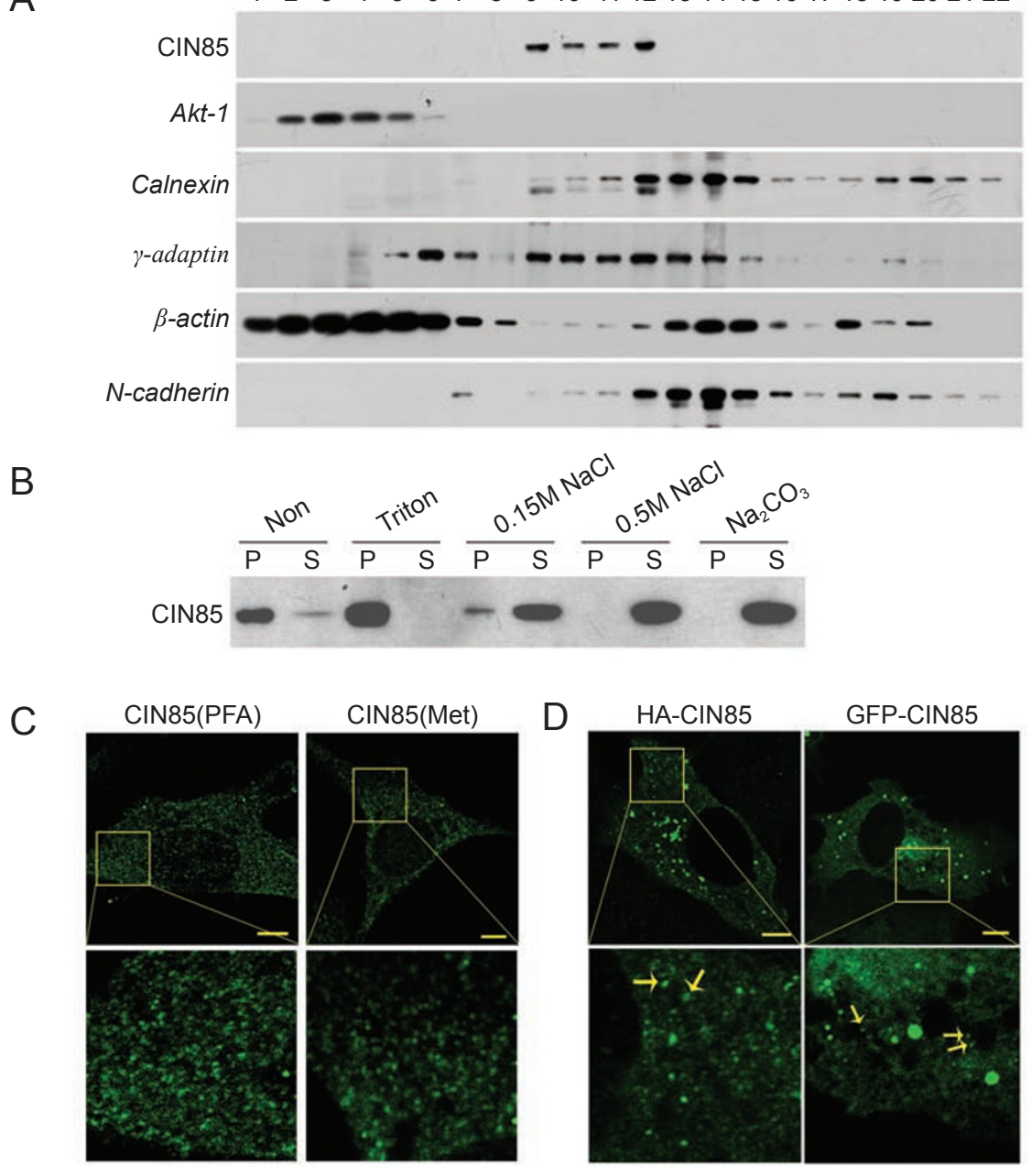

$\mathrm{E}$
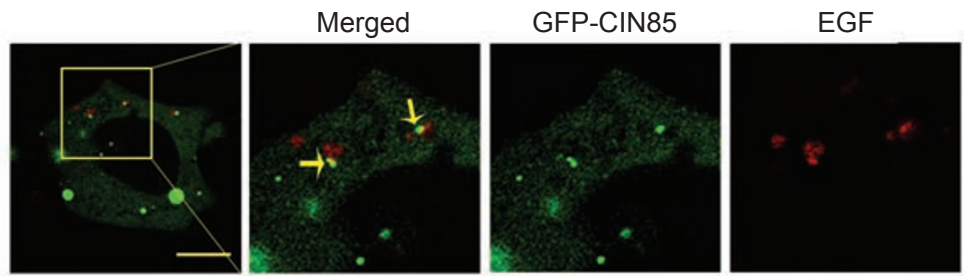

Figure 1 CIN85 located on the cellular membrane. The scale bar is $10 \mu \mathrm{m}$. (A) Subcellular membrane fractionation by noncontinuous sucrose density gradient centrifugation to evaluate endogenous CIN85 localization. 1, 2, ..., 22 are fractions from sucrose gradient centrifugation, with 1 being at the top and 22 at the bottom. Akt-1, cytoplasmic protein marker; calnexin, ER marker; $\gamma$-adaptin, Golgi apparatus marker; $\beta$-actin, cytoskeleton marker; $N$-cadherin, plasma membrane marker. (B) Association of CIN85 with the inner membrane. The supernatant obtained following centrifugation at $12000 \mathrm{~g}$ was further pelleted at $200000 \mathrm{~g}$ for $60 \mathrm{~min}$. $P$, pellet following $200000 \mathrm{~g}$ centrifugation; S, supernatant following $200000 \mathrm{~g}$ centrifugation. Non: lysate buffer, Triton/0.15 $\mathrm{M} \mathrm{NaCl} / 0.5 \mathrm{M} \mathrm{NaCl} / \mathrm{Na}_{2} \mathrm{CO}_{3}$ : lysate buffer with addition of the indicated reagent, $1 \%$ Triton/0.15 $\mathrm{M}$ $\mathrm{NaCl} / 0.5 \mathrm{M} \mathrm{NaCl} / 25 \mathrm{mM} \mathrm{Na}_{2} \mathrm{CO}_{3}$, respectively. (C) Immunofluorescence staining of endogenous CIN85 in 3T3-L1 cells. Cells were fixed with methanol (Met) or 4\% PFA, and immunostained with mAb against CIN85. The lower panel is a magnified view of the upper panel in the block. (D) Localization of exogenous CIN85 in COS-7 cells. COS-7 cells transfected with HA-CIN85 or GFP-CIN85 were fixed with PFA and immunostained with anti-HA antibody or directly subjected to imaging. The lower panel is a magnified view of the upper panel in the block. Arrows indicate that CIN85 was located on the edges of some dark circular areas. (E) The localization of CIN85 on the edges of endosomes containing EGF. Alexa Fluor-labeled EGF (red) was allowed to undergo endocytosis in GFP-CIN85-transfected COS-7 cells and was observed with time-lapse confocal microscopy. One picture is shown. The left panel in the block is enlarged in the three right panels. Arrows indicate that CIN85 was located on the edges of some endosomes containing EGF. 
A

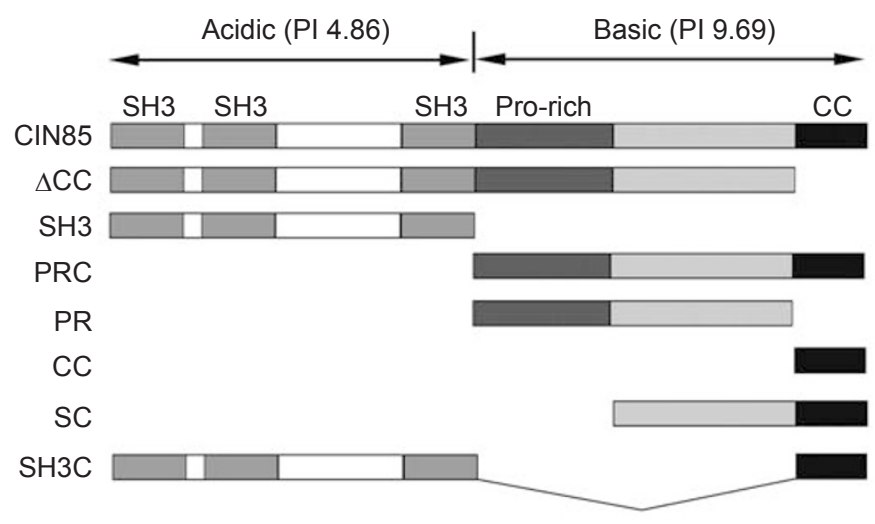

B

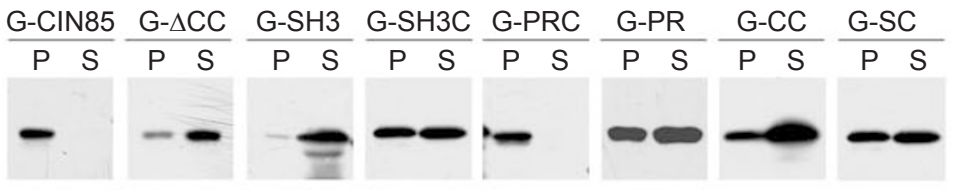

C

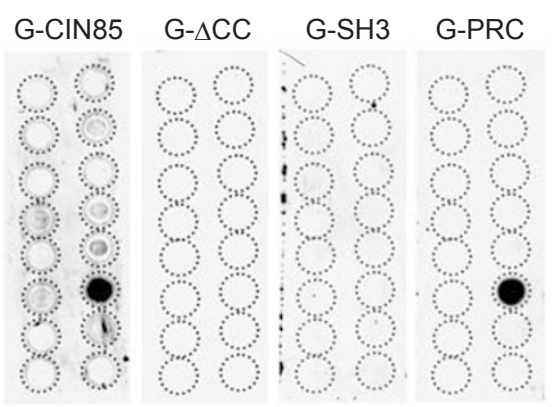

His-SH3 His-PRC

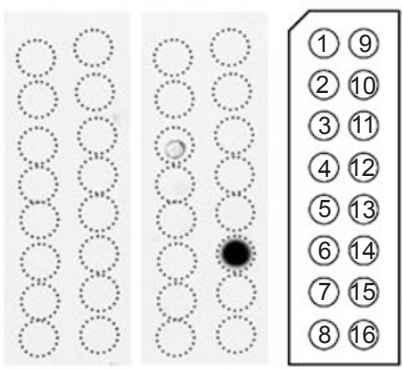

$\mathrm{D}$
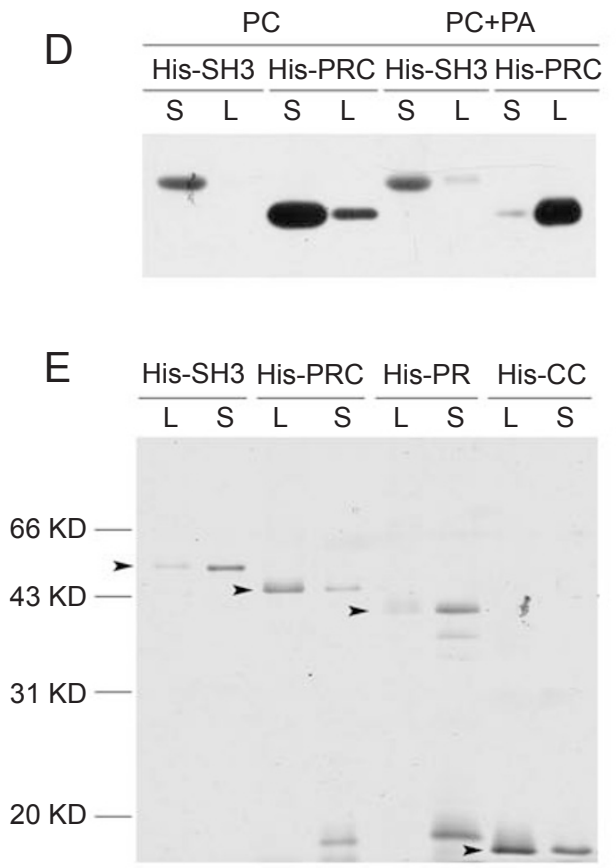

Figure 2 The coiled-coil domain of CIN85 regulated the membrane and phosphatidic acid association. (A) Construction of truncated forms of CIN85. (B) The association of exogenous eGFP-tagged truncated forms of CIN85 with vesicular membranes. $P$, pellet of $200000 \mathrm{~g}$ centrifugation; S, supernatant of $200000 \mathrm{~g}$ centrifugation. (C) Interaction between CIN85 and phosphatidic acid on the PIP strip membrane. Truncated forms of G-CIN85, G- $\triangle C C$, G-SH3 and G-PRC, eGFP-tagged CIN85 were expressed and purified from HEK293 cells. His-SH3 and His-PRC indicate His-tagged SH3 domains and the PRC fragment purified from E. coli. The phospholipids on the membrane are: 1, lysophosphatidic acid; 2, lysophosphatidylcholine; 3, phosphatidylinositol; 4, Ptdlns(3)P; 5, Ptdlns(4)P; 6, Ptdlns(5)P; 7, phosphatidylethanolamine; 8, phosphatidylcholine; 9 , sphingosine 1-phosphate; 10, Ptdlns(3,4)P; 11, Ptdlns(3,5)P; 12, Ptdlns(4,5)P; 13, Ptdlns(3,4,5)P; 14, phosphatidic acid; 15, phosphatidylserine; 16, blank. (D) Association of His-PRC with liposomes of phosphatidyl choline and phosphatidic acid (90\% PC and $10 \%$ PA). PC, liposomes of phosphatidyl choline; $P C+P A$, liposomes of phosphatidyl choline and phosphatidic acid; $S$, soluble fraction; $L$, liposome fraction. Samples were western blotted with anti-His antibody. (E) Association of His-PRC and His-CC with liposomes of phosphatidyl choline and phosphatidic acid ( $90 \%$ PC and 10\% PA). Purified His-tagged protein was incubated with PA-containing liposome. $S$, soluble fraction; $L$, liposome fraction. 
phosphatidic acid, a fusogenic lipid. The interaction with phosphatidic acid was specific because CIN85 did not bind the same ionic charged lysophosphatidic acid, phosphatidylinositol phosphates, sphingosine phosphate or phosphatidylserine (Figure 2C).

Consistent with the differential centrifugation results, the eGFP- $\triangle \mathrm{CC}$ fusion protein could no longer bind to phosphatidic acid following deletion of its coiled-coil domain, whereas the C-terminus of CIN85, which contains the coiled-coil domain, the linker region and the prolinerich region (PRC fragment), retained the phosphatidic acid-binding ability (Figure 2C). His-tagged CIN85 Cterminus (His-PRC) purified from E. coli was also shown to bind to phosphatidic acid, but His-tagged N-terminus (His-SH3) was not able to bind (Figure 2C).

To verify the membrane blot results, His-SH3 or HisPRC fusion protein was incubated with liposomes made of phosphatidyl choline or both phosphatidyl choline and phosphatidic acid (10\% PA and 90\% PC). There was little association of His-SH3 with either type of liposomes (Figure 2D). However, His-PRC could specifically associate with liposomes of phosphatidyl choline and phosphatidic acid, but there was little association with liposomes of phosphatidyl choline (Figure 2D).

Further incubation of His-PR or His-CC with phosphatidic acid-containing liposomes (10\% PA and 90\% PC) showed that PR did not interact with the liposomes, while His-CC had association with the liposomes, although the interaction was weaker than with His-PRC (Figure 2E). Thus, our data indicate that the coiled-coil domain contains at least part of the phosphatidic acidbinding motif.

Deletion of the coiled-coil domain reduces EGFR downregulation and CIN85 interaction with $\mathrm{Cbl}$

Since most of CIN85's effectors are associated with its SH3 domains or its proline-rich domain, and the coiledcoil domain participates mainly in oligomerization, the mutant CIN85 lacking the coiled-coil domain can be a powerful tool to study the importance of CIN85 membrane association. Although the SH3 domains of CIN85 may potentially interact with the proline-rich region within CIN85, no intramolecular interactions between the SH3 domains and the proline-rich region were observed (Supplementary information, Figure S2). This finding is consistent with the observation of Daniela Jozic et al. that CIN85 has a very low level of interaction with the three $\mathrm{SH} 3$ domains [20].

It is well known that CIN85 mediates downregulation of activated EGFR from the cell surface by interacting with $\mathrm{c}-\mathrm{Cbl}$ and endophilins $[1,4,5,10-12]$. The effect of coiled-coil domain deletion from CIN85 on EGF-stimu- lated EGFR downregulation was examined by measuring EGFR endocytosis and degradation in COS-7 cells expressing eGFP-tagged CIN85 fusion proteins.

Transient expression of eGFP- $\triangle \mathrm{CC}$ in COS-7 cells retarded the downregulation of EGFR (Figure 3A). Interaction of CIN85 with EGFR-associated c-Cbl is the initial step in EGFR endocytosis; and relays on the SH3 domains of CIN85 and the proline-rich motif of $\mathrm{c}-\mathrm{Cbl}[1$, $4,10]$. However, this interaction was greatly reduced in the absence of the coiled-coil domain, which itself does not interact with c-Cbl (Figure 3C and 3D).

EGFR endocytosis was analyzed by FACS as described in Materials and methods. EGF-stimulated EGFR internalization was greatly inhibited in COS-7 cells expressing eGFP- $\triangle \mathrm{CC}$, compared with COS-7 cells expressing eGFP or eGFP-CIN85 wild type (eGFP-CIN85) (Figure 3B).

In addition, EGF was found to be concentrated in the perinuclear region in cells that had a moderate GFP$\Delta \mathrm{CC}$ expression level after a 180 -min incubation (Figure $3 \mathrm{E}$, left panels), which is distinct from its degradation in non-transfected cells and in eGFP-CIN85-positive cells (Figure 3E, right panels). The EGF-rich perinuclear region can be stained with filipin, which labels cholesterol. Cholesterol is a well-characterized endosomal marker that is localized mainly in the MVB compartment in the endocytic pathway (Supplementary information, Figure S3) [21]. This result indicates that $\triangle C C$ might function as a dominant-negative mutant that blocks the EGF sorting process.

CIN85 co-localizes with EGF-containing vesicles after $E G F$ endocytosis and is involved in EGF degradation

Considering the important role that CIN85 plays in EGF downregulation, we explored whether CIN85 was present on the endosomal membrane. Alexa Fluor-EGF incubated cells were pulse-chased for different time (Figure 4A). We found that CIN85 was recruited onto the EGF-containing vesicles after endocytosis. Co-localization was faintly visible after a 7.5-min EGF chase at the periplasma membrane region with very small EGF vesicles (Figure 4A, upper panel), while it gradually increased until the internalized EGF was concentrated in the perinuclear region (Figure 4A, second and third panels). The co-localization was gradually lost over time (Figure 4A, fourth panel).

This dynamic process was also shown with confocal time-lapse microscopy in living cells (Figure 5A and 5B). The localization of CIN85 on the edge of the round dark areas was especially obvious in cells with a low CIN85 expression level, which did not contain visible large dotlike structures (Figure 5A). Notably, in a few cells that 
A

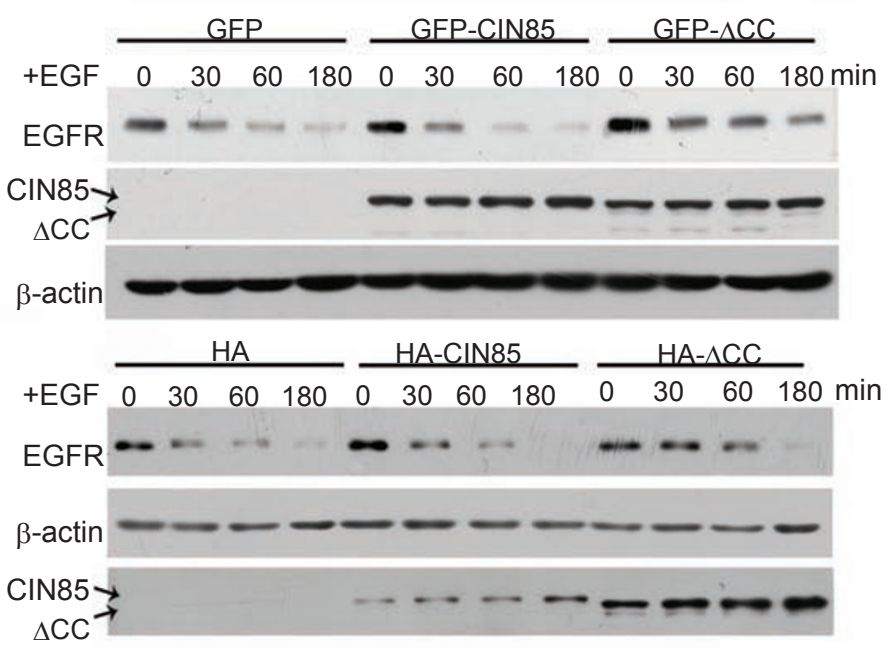

C

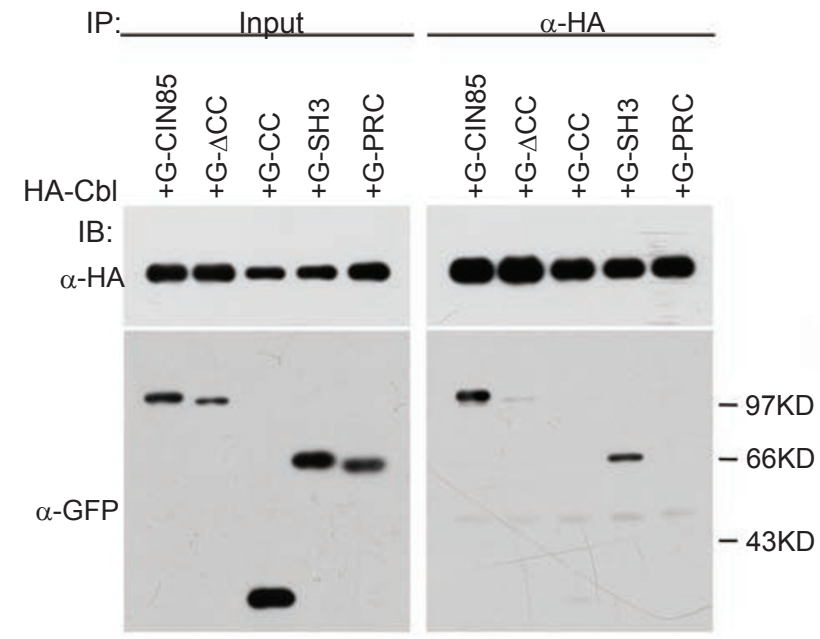

B

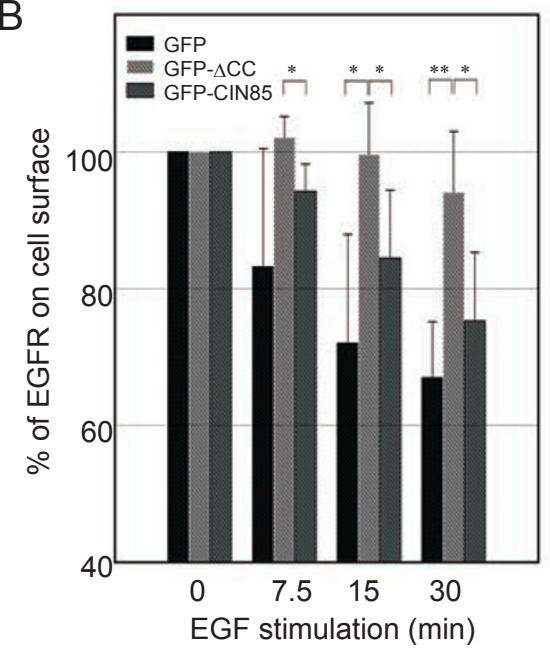

D

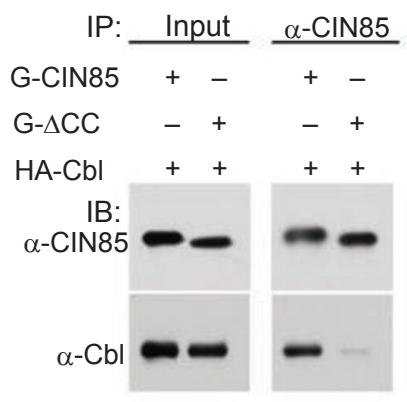

E

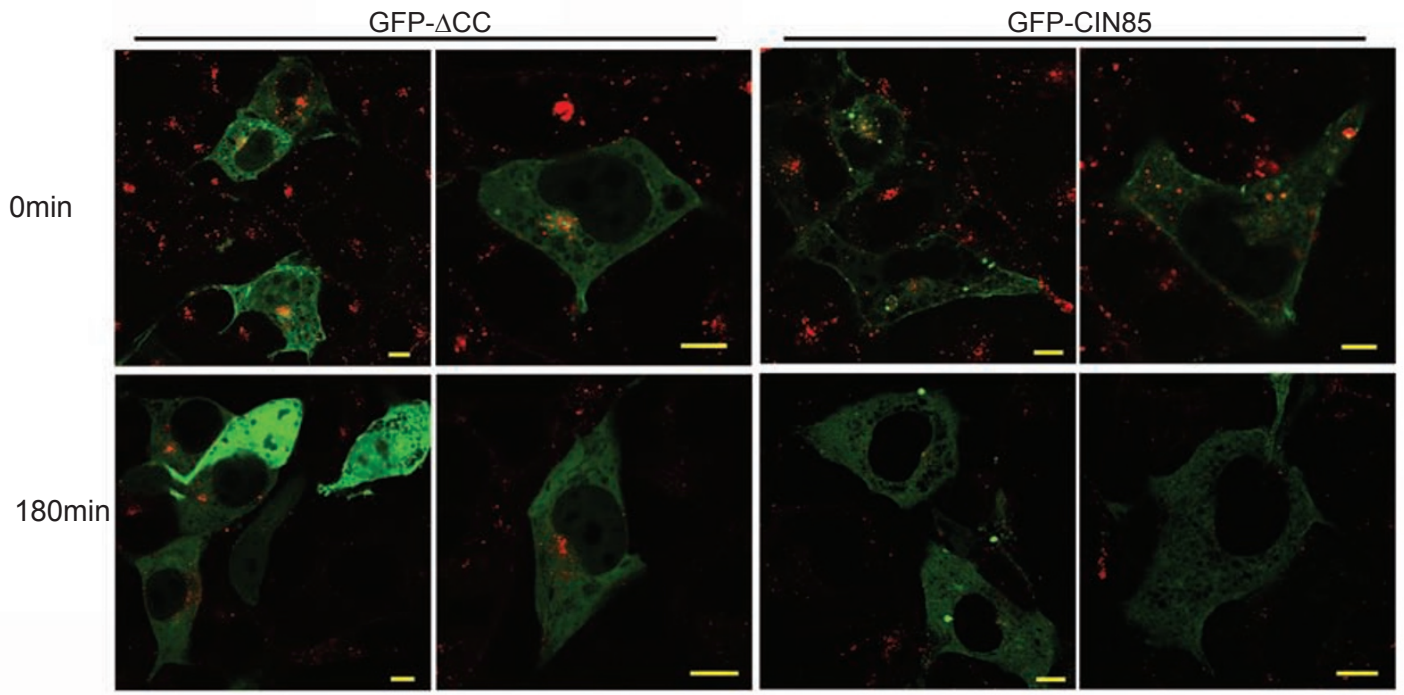


had very large dot-like structures, the EGF endocytosis rate was somewhat reduced (data not shown), which may account for the slight retardation of EGF endocytosis observed in Figure 3B.

Coiled-coil domain deletion impaired the recruitment of CIN85 onto EGF-positive vesicles (Figure 5C, supplementary information, video S3). The endocytosis rate was much slower in cells transfected with GFP$\triangle \mathrm{CC}$ (Figure 5C, supplementary information, video S3). In cells that had lower $\triangle \mathrm{CC}$ expression, the endocytosis seemed normal and EGF could concentrate in the perinuclear region (data not shown).

The dynamic recruitment of CIN85 by EGF-containing vesicles and the EGF degradation blockage effect of GFP- $\triangle C C$ reminded us that CIN85 may also be involved in the EGF trafficking and sorting process after EGF endocytosis. Therefore, we evaluated the EGF degradation in CIN85 knockdown cells. The knockdown effect was shown in Figure 4B. Silencing of CIN85 greatly blocked the endocytosis of EGF, whereas transferrin internalization was normal (Supplementary information, Figure S4). Alexa Fluor labeled EGF was pulse-chased and released for different time in CIN85 knockdown or negative control cells. Although less EGF was internalized in CIN85 knockdown cells, there was more EGF left undegraded after long-time release (Figure 4C).

\section{CIN85 locates on a specific endosomal compartment re- lated to ESCRT formation \\ Exogenous expression of CIN85 in COS-7, Hela or}

HEK293 cells caused the formation of vesicle-like structures $[4,14,22]$, and the coiled-coil-deleted fusion protein lost this vesicular expression pattern (Supplementary information, Figure S5A).

Double immunofluorescence staining showed partial co-localization of CIN85 with HRS, but not with M6-PR (a late endosome marker) or with EEA1 (early endosome marker) (Supplementary information, Figure S5B). As a protein component in ESCRT-0, HRS is at the core of the ESCRT complex, which controls MVB formation in endosomes [23, 24]. This observation suggests that CIN85 is located on the specific endosomal compartment involved in cargo sorting in the MVB pathway and dissociates before lysosome fusion.

Vps4, an ATPase that regulates ESCRT complex dissociation from the endosomal membrane, is a cytoplasmic protein and is recruited to ESCRT complex III on the endosomal membrane in the presence of ATP [24, 25]. An ATPase-negative Vps4 mutant, which binds but does not hydrolyze ATP, constitutively associates with endosomal membranes and forms the "E class" structure $[25,26]$. Wild-type Vps4 did not co-localize with CIN85 (Figure 6C), while the dominant-negative form of Vps4 (Vps4 M) co-localized with CIN85 on the enlarged endosomal membranes (Figure 6D). CIN85 also co-localized with another ESCRT complex III protein, CHMP4B (Figure 6E), which also accumulated on the enlarged endosomal membrane when fused to a fluor-protein tag $[19,24]$. This behavior suggests that CIN85 is involved in the ESCRT pathway. Interestingly, when co-expressed

Figure 3 Epidermal growth factor receptor (EGFR) downregulation was reduced by deletion of coiled-coil domain. The scale bar is $10 \mu \mathrm{m}$ (A) EGFR downregulation in $\triangle$ CC-transfected COS-7 cells was inhibited. COS-7 cells transfected with GFPor HA-tagged $\mathrm{CIN} 85 / \triangle \mathrm{CC}$ or mock vector were stimulated by EGF $25 \mathrm{ng} / \mathrm{ml}(+E G F)$. At the indicated time $(0,30,60,180$ $\mathrm{min}$ ), cells were harvested for immunoblotting of EGF receptor (EGFR). Actin (actin) was used as the loading control. (B) Reduced EGFR endocytosis in COS-7 cells expressing eGFP- $\triangle$ CC fusion protein. Cells were double-sorted by EGFR and eGFP fluorescence as per the methods suggested. EGFR was immuno-labeled by PE-conjugated antibody and eGFP was tagged to CIN85 or $\triangle \mathrm{CC}$. PE fluorescence intensity in eGFP-positive cells was analyzed with the Flowjo program. The PE fluorescence intensity in COS-7 cells without EGF stimulation was normalized as $100 \%$ EGFR level. The results were the average of four independent experiments. GFP, COS-7 cells expressing eGFP protein. CIN, COS-7 cells expressing eGFPCIN85 fusion protein. $\triangle C C$, COS-7 cells expressing eGFP- $\triangle C C$ fusion protein. Student's $t$-test was performed. The $P$ value between eGFP-expressing cells and eGFP- $\triangle$ CC-expressing cells was 0.074 (7.5 min); *0.020 (15 min); and **0.004 (30 $\mathrm{min})$, respectively. For the samples between eGFP-expressing cells and eGFP-CIN85-expressing cells, the $P$ value was 0.337 (7.5 $\mathrm{min}) ; 0.232$ (15 $\mathrm{min})$; and 0.245 (30 $\mathrm{min})$, respectively. For the samples between eGFP-CIN85-expressing cells and eGFP- $\Delta$ CC-expressing cells, the $P$ value was $* 0.030$ (7.5 min); $* 0.048$ (15 min); and *0.031 (30 min), respectively. (C) Evaluation of the interaction between CIN85 or its truncated forms and C-Cbl. HA-Cbl co-expressed with eGFP-tagged CIN85 or its truncated forms in HEK293 cells was immunoprecipitated $(I P)$ by anti-HA antibody $(\alpha-H A)$. The input and immunoprecipitates were immunoblotted $(I B)$ by anti-HA antibody $(\alpha-H A)$ or anti-eGFP antibody $(\alpha-G F P)$. The numbers indicated the sizes of the molecular weight markers. (D) Co-immunoprecipitation of C-Cbl by anti-CIN85 antibody. In HEK293 cells co-expressing HA$\mathrm{Cbl}$ with eGFP-CIN85 (G-CIN85) or eGFP- $\triangle \mathrm{CC}(\mathrm{G}-\Delta C C)$, the CIN85 fusion proteins were immunoprecipitated by anti-CIN85 antibody $(\alpha-C / N 85)$. The input and immunoprecipitated samples were immunoblotted with anti-c-Cbl antibody ( $\alpha$-Cb/) or antiCIN85 ( $\alpha$-CIN85) antibody. (E) The degradation was blocked in coiled-coil domain-deleted mutant-transfected cells. COS-7 cells transfected with GFP-CIN85 or GFP- $\triangle$ CC were internalized during incubation with Alexa Fluor-647 EGF (red) for 60 min, washed with binding buffer, and then fixed immediately or after incubation in binding buffer for 180 min. 
A

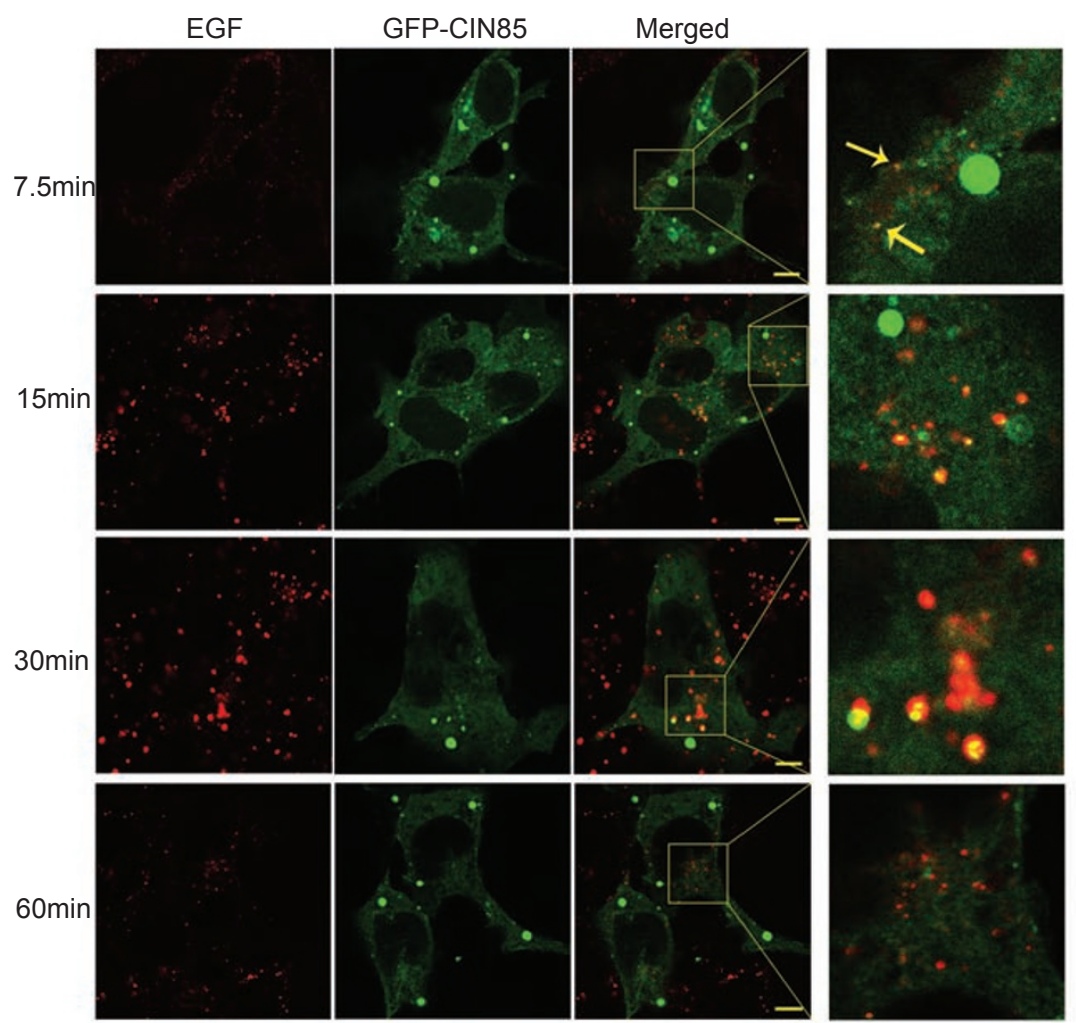

B

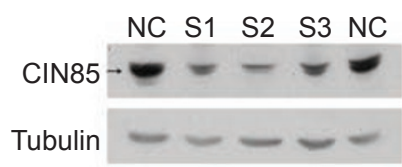

C

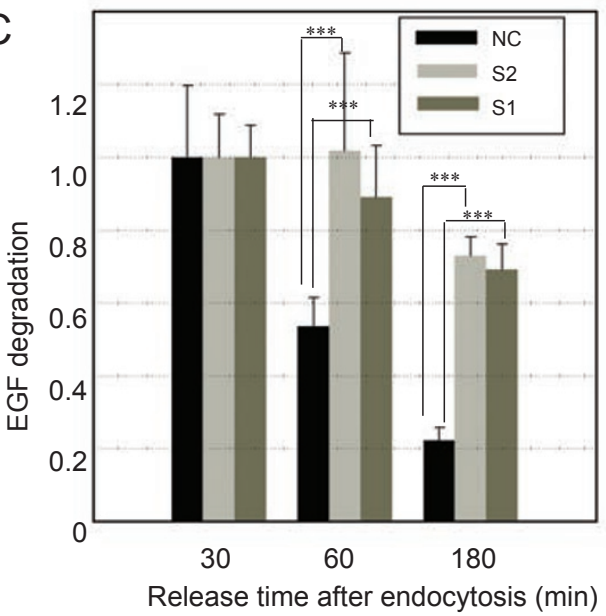

Figure 4 CIN85 co-localized with EGF-containing vesicles after EGF endocytosis and was involved in the degradation of EGF. The scale bar is $10 \mu \mathrm{m}$. (A) CIN85 co-localized with the Alexa fluor-labeled EGF after endocytosis. COS-7 cells transfected with GFP-CIN85 were incubated with Alexa fluor-647-EGF for $30 \mathrm{~min}$ on ice and then transferred to a $37^{\circ} \mathrm{C}$ incubator for 7.5, 15, or $30 \mathrm{~min}$. The right panel is a magnified view of the third panel in the block. (B) Knockdown efficiency of CIN85 siRNA transfection. COS-7 cells were transfected with three CIN85 siRNA (S1, S2, S3) or a negative ctrl sequence (NC) for $72 \mathrm{~h}$ and immunoblotted with CIN85 antibody. Tubulin was used as a loading ctrl. (C) Silencing of CIN85 expression blocked EGF degradation. COS-7 cells transfected with CIN85 siRNA $(S 1, S 2)$ or the negative ctrl sequence $(N C)$ were pulse-chased with Alexa fluor-555-EGF, and released for 30,60, or 180 min after washing with binding buffer. The cells were permeabilized with $0.05 \%$ saponin before fixation with $4 \%$ PFA. Images were acquired with wide-field confocal microscopy using identical exposure settings and the average EGF fluorescence intensities were calculated for each view field (20 fields per sample). These values were normalized to the average value observed following $30 \mathrm{~min}$ of release. The experiments were repeated three times, and the reported values are the means + SEM of a representative experiment. The $P$ values of the comparisons between NC and S1 were ***7.10E-06 for $60 \mathrm{~min}$ and ***3.21E-12 for $180 \mathrm{~min}$; between NC and S2, ***4.89E-05 for 60 min and $* * * 2.20 \mathrm{E}-14$ for $180 \mathrm{~min}$; and between $\mathrm{S} 1$ and S2, 0.18 for $60 \mathrm{~min}$ and 0.16 for $180 \mathrm{~min}$. 
A
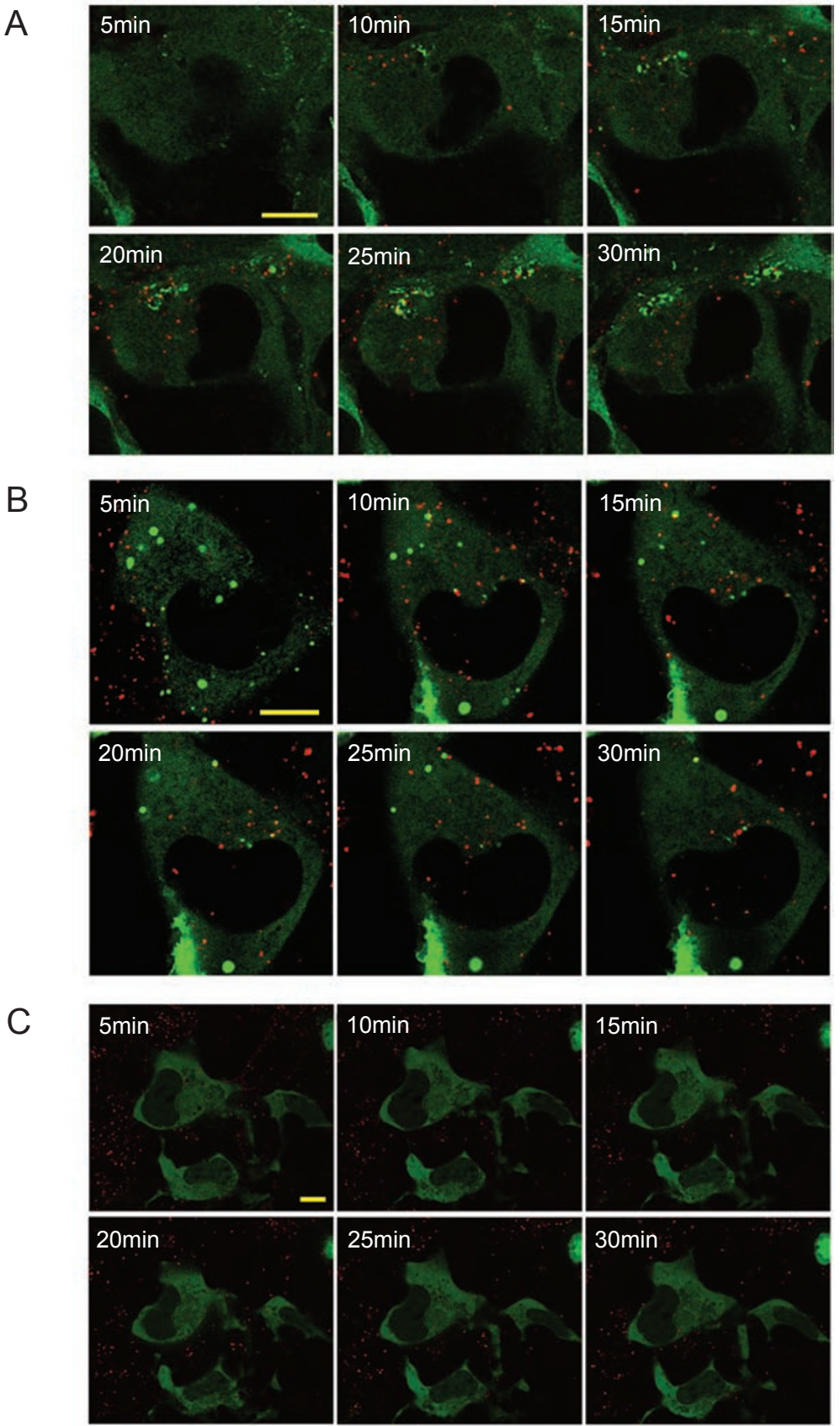

Figure 5 EGF endocytosis analysis in GFP-CIN85/ACC-transfected living cells by confocal time-lapse microscopy. The scale bar is $10 \mu \mathrm{m}$. (A) GFP-CIN85-transfected COS-7 cells were labeled with EGF-647 for 30 min on ice, washed with $37{ }^{\circ} \mathrm{C}$ binding buffer, incubated in binding buffer and imaged by live video microscopy from 5 min after the initial $37^{\circ} \mathrm{C}$ incubation (Supplementary information, Video S1 in the block). Pictures are shown for 5-min intervals. (B) GFP-CIN85-transfected COS-7 cells were labeled and released as in (A), pictures are similarly shown for 5-min intervals (Supplementary information, Video S2). (C) GFP- $\triangle$ CC-transfected COS-7 cells were labeled and released as in (A). Pictures are similarly shown for 5-min intervals (Supplementary information, Video S3).

with Vps4 M or CHMP4B, both CIN85 and $\triangle \mathrm{CC}$ were found to cluster and partially overlap with these proteins (Figure 6D and 6E). Possible protein interactions between CIN85 and Vps4 or CHMP4B were then analyzed.
The Vps4 dominant-negative form (Vps4 M) was coprecipitated by CIN85, while wild-type Vps4 had a much weaker interaction with CIN85 (Figure 6A). Notably, full-length CIN85 and the $\triangle \mathrm{CC}$ mutant were similarly 
A

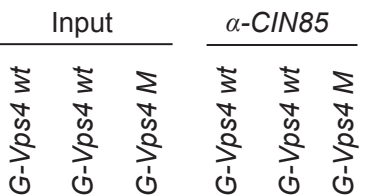

GFP-CIN85

-

$-$

IB: $\alpha-C / N 85$ $\alpha-G F P$

$$
-2
$$

(-GFP

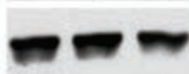

B

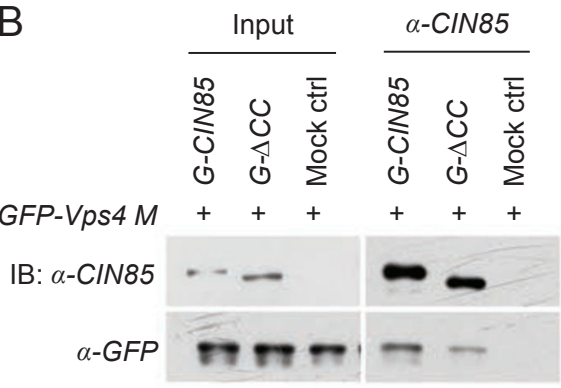

C
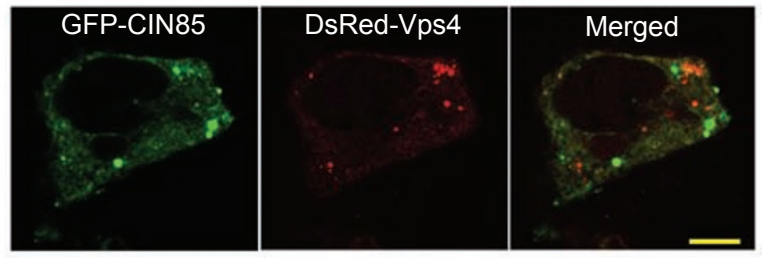

D
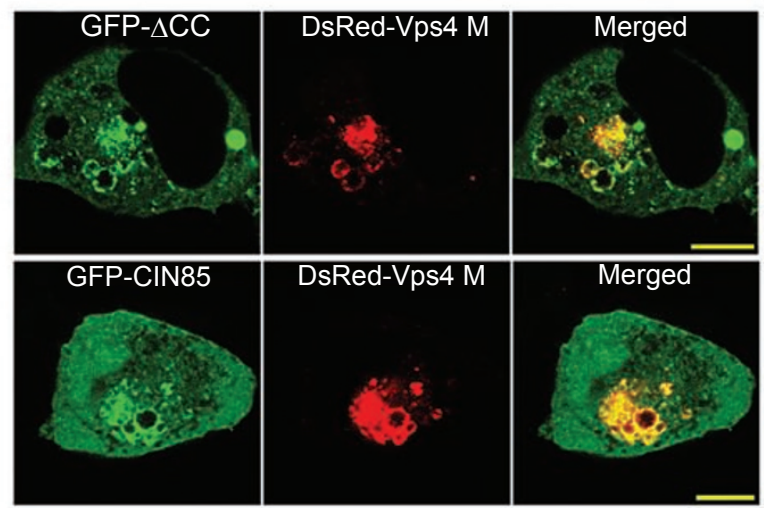

E
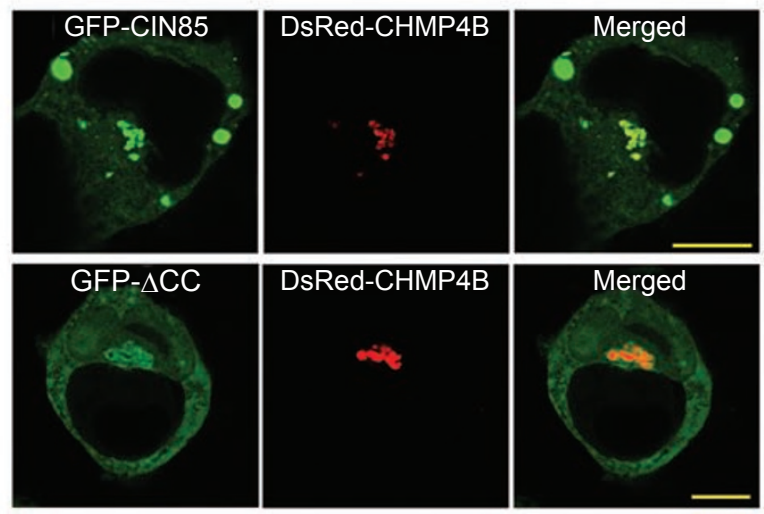

Figure 6 Recruitment of CIN85 onto the E class structure. The scale bar is $10 \mu \mathrm{m}$. (A) Interaction of CIN85 with the dominantnegative form, but not wild-type form of Vps4. Lysates from HEK293 cells co-expressing GFP-CIN85(+)/mock vector(-) and GFP-tagged Vps4 (G-Vps4 wt) or the ATPase dominantnegative form (G-Vps4 $M$ ) were subjected to immunoprecipitation with anti-CIN85 antibody $(\alpha-C / N 85)$. The input and immunoprecipitates were immunoblotted with anti-CIN85 antibody $(\alpha-C I N 85)$ and anti-eGFP antibody $(\alpha-G F P)$. (B) Evaluation of the interactions of both CIN85 and $\triangle \mathrm{CC}$ with the GFP-Vps4 mutant. Lysates from HEK293 cells co-expressing CIN85 (GFPCIN85)/ $\triangle C C$ (GFP- $\triangle C C$ )/mock vector and GFP-tagged Vps4 mutant (G-Vps4 M) were subjected to immunoprecipitation (IP) with anti-CIN85 antibody $(\alpha-C / N 85)$. The input and immunoprecipitates were immunoblotted (IB) with anti-CIN85 antibody $(\alpha-C I N 85)$ and anti-GFP antibody $(\alpha-G F P)$. (C) CIN85 had no co-localization with Vps4 wild type. COS-7 cells co-transfected with eGFP-tagged CIN85 and DsRed-tagged Vps4 wild type were observed directly after fixation with $4 \%$ PFA. (D) Co-localization of GFP-tagged CIN85 fusion protein (GFP-CIN85/ACC) with DsRed-tagged Vps4 mutant (Vps4 M). GFP fusion proteins were co-expressed with Vps4 M in HEK293 cells. The cells were fixed with $4 \%$ PFA and observed by confocal microscopy directly. (E) Co-localization of GFP-tagged CIN85 fusion protein (GFPCIN85/ACC) with DsRed-tagged CHMP4B. GFP-tagged CIN85 fusion protein (GFP-CIN85/ $\triangle C C$ ) was co-expressed with DsRedtagged CHAMP4B in HEK293 cells. Cells were fixed with 4\% PFA and observed by confocal microscopy.

co-precipitated with the Vps4 mutant (Vps4 M) (Figure $6 \mathrm{~B})$. However, CHMP4B was not detected in the immunoprecipitates (data not shown).

\section{Discussion}

Our findings clearly show that CIN85 specifically and directly interacts with phosphatidic acid (Figures 1 and 2). Phosphatidic acid is a tightly controlled fusogenic lipid in the membrane. It is generated from non-fusogenic phosphatidyl choline by phospholipase D (PLD), an enzyme that is activated in response to extracellular signal molecules $[27,28]$. Phosphatidic acid regulates vesicular trafficking in endocytosis and secretion by altering lipid bilayer properties and facilitating vesicle targeting, budding and fusion [27-29]. Endophilin, which interacts with CIN85, is an enzyme that converts lysophosphatidic acid into phosphatidic acid [12]. EGFR downregulation is a typical extracellular signal-induced membrane trafficking process $[6,8,9]$. In response to EGF stimulation, PLD is activated to generate phosphatidic acid in the plasma membrane and its inhibition blocks receptor endocytosis $[28,30,31]$. Two PLD isoforms, PLD1a and 1b, were also found to be located on the endosomal compartment $[32,33]$. Therefore, the phosphatidic acid present in the 
plasma membrane and endosomal membrane may be an essential component for recruitment of CIN85 to the activated EGFR in endocytosis and the sorting process. It is reasonable to speculate that CIN85 couples protein endocytosis and cargo sorting to fusogenic phosphatidic acid, which may facilitate membrane budding and fusion during RTK downregulation.

The mechanism by which CIN85 interacts with phosphatidic acid is complex. The positive charge of the C-terminus plays an important role in this interaction, because increases in the $\mathrm{pH}$ or ionic concentration eliminate both the phosphatidic acid and membrane association (Figure 1B and our unpublished data). The coiledcoil domain is important for both proper localization and the phosphatidic acid-binding ability of CIN85. We found that the coiled-coil domain of CIN85 directly interacts with phosphatidic acid. Deletion of the coiled-coil domain eliminated both the association between CIN85 and phosphatidic acid and the fractionation of CIN85 with the membrane fraction (Figure 2C-2E). Inclusion of the proline-rich domain and the linker region enhanced the fractionation of CIN85 with the membrane fraction and its interaction with phosphatidic acid, although these domains did not associate with phosphatidic acid by themselves. We hypothesize that the full phosphatidic acid-binding motif spans both the coiled-coil domain and the proline-rich domain. Only when the molecule's Cterminus was oligomerized did a correct binding module form. Similar to the FYVE module in the HRS protein or $\mathrm{PH}$ module in the dynamin-1, pleckstrin, etc., the ability of the coiled-coil domain to bind phospholipids is necessary but not sufficient for membrane association. The proline-rich region and linker region may facilitate membrane localization by interacting with other proteins.

Aside from facilitating RTK endocytosis, the associations of CIN85 with the membrane and phosphatidic acid should be of great importance for its participation in endosomal trafficking and sorting. Our results show that CIN85 dynamically co-localizes with EGF-containing endosomes after endocytosis (Figures 4 and 5), and that EGF degradation is retarded following knockdown of CIN85 expression (Figure 4D). These findings indicate that CIN85 has a role in the cargo-sorting process. Membrane-associated Vps4 could act as an effector that recruits CIN85 (Figure 6), although additional intervening components may also be involved. Expression of the dominant-negative form of Vps4 or fluor-protein-tagged CHMP4 (snf7) stabilized the membrane-associated state of endosomal sorting-required complex proteins and led to formation of enlarged tabulated endosomal compartments (termed "class E" structures) [19, 24-26]. When co-expressed with a Vps4 mutant, a significant portion of
CIN85 became associated with the aberrant endosomal membrane, which suggests that CIN85 is at least transiently bound to the cargo-sorting endosomal membrane. Therefore, CIN85 may also signal for the assembly of the ESCRT complex on the endosomal membrane (Figure 6). This hypothesis is consistent with the discovery that CD2AP, a CIN85 family member, co-localizes with a specific Rab4-labeled endosomal compartment, but does not co-localize with EEA1- or Rab11-labeled endosomes $[34,35]$. The observed interaction of Alix, a MVB-specific LBPA (lysobisphosphatidic acid)-binding protein, with CIN85 may provide additional support for a role of CIN85 in the endosome-sorting process [36, 37]( Supplementary information, Figure S1).

In temporal order, CIN85, which associates with the receptor after endocytosis, may initiate ESCRT assembly on endosomes. As the CIN85-associated vesicular membrane contains both phosphatidic acid and EGFR, assembly of the ESCRT complex around CIN85 can induce membrane budding and fusion during MVB formation and sort the receptor in the MVBs. In this sense, CIN85 not only mediates EGFR endocytosis but also regulates the receptor-sorting process. This study provides evidence suggesting that CIN85 may function as a scaffold protein, which integrates endocytosis and cargo-sorting pathways through its membrane localization and phosphatidic acid interaction. The observation of retardation of EGF degradation in CIN85 knockdown cells strongly supports our hypothesis (Figure 4D).

Deletion of the coiled-coil domain in CIN85 diminishes the membrane localization and phosphatidic acid-binding ability of CIN85 and blocks the downregulation of EGFR that is mediated by CIN85 (Figures 2 and 3). Both recruitment of CIN 85 by c-Cbl and the association of CIN85 with endocytic vesicles require the presence of the coiled-coil domain (Figure 3). The coiled-coil domain in CIN85 is known to be required for interaction between CIN85 molecules (Supplementary information, Figure S2). The coiled-coil fold is present in many endocytic proteins, such as Hrs, epsins, Eps15, sorting nexins and others. Similarly, deletion of the coiled-coil domains in Hrs and sorting nexin 1 blocks the formation of endocytic vesicles upon over-expression of the mutant protein $[6,13]$.

Given the important role that the coiled-coil domain plays in heterotypic and homotypic oligomerization, it is difficult to attribute the blockage of EGFR downregulation observed for $\triangle \mathrm{CC}$ directly to the loss of interaction with phosphatidic acid and the membrane. However, the association of CIN85 as a scaffold protein with the endosomal membrane increases the local concentration of CIN85-anchored signaling complexes in the proximity of 
activated receptors, which may provide spatial and temporal co-ordination of critical steps in receptor endocytosis. Nevertheless, further work is required to clarify the exact relationship between CIN85 membrane localization and EGFR downregulation. For example, we suggest that future studies aim at identifying and characterizing CIN85 point mutants that are unable to bind PA.

CIN85, but not $\triangle \mathrm{CC}$, can recruit c-Cbl, while both $\mathrm{CIN} 85$ and $\triangle \mathrm{CC}$ can cluster onto the enlarged endosomal membrane formed in the context of Vps4 M or CHMP4B over-expression (Figure 6D and 6E). EGF was found to accumulate in the perinuclear (MVB) region after a 180min period of degradation in $\triangle \mathrm{CC}$-transfected COS-7 cells (Figure $3 \mathrm{E}$ ). In some cells, $\triangle \mathrm{CC}$ clearly concentrated as a dot-like structure in the perinuclear region, where EGF accumulated (Figure 3E and Supplementary information, Figure S3). These results indicate that $\triangle \mathrm{CC}$ might function as a dominant-negative mutant during the cargo-sorting process. Nevertheless, further study is needed to explore the roles of CIN85 in the MVB-sorting process and to identify the recruited effectors.

\section{Materials and Methods}

\section{Materials}

Anti-CIN85 (05-733) monoclonal antibody was purchased from Upstate. Anti-eGFP, anti-Akt, anti-EGFR, anti-Cbl, anti-HRS, anti- $\gamma$-adaptin and anti-calnexin antibodies were bought from Santa Cruz. Anti-Flag and anti-HA monoclonal antibodies and horse radish peroxidase-conjugate secondary antibodies were ordered from Sigma. Anti-N-cadherin was from BD. Anti-M6PR was from Abcam. Alexa Fluor 555/647 EGF, PIP Strips ${ }^{\mathrm{TM}}$ membrane and secondary antibodies (Alexa Fluor 488/546/647 conjugates) for immunofluorescence were the products of Molecular Probes. Phosphatidic acid and phosphatidylcholine were both from Sigma. CIN85 siRNA were synthesized by Shanghai GenePharma Co. Ltd. The Leica laser scanning confocal microsystem, including the Leica TCS SP2 confocal microscope, Leica confocal scanner, and Leica confocal acquisition software, was used with the HCX PL APO 1bd. BL 63.0X/1.4 oil objective at 1.4 numerical aperture at a working temperature of $22{ }^{\circ} \mathrm{C}$. For live video confocal analysis, Leica TCS SP5 confocal microscope was used.

\section{Plasmid construction and protein expression}

Mutants of mouse CIN85 were constructed by PCR amplification and tagged with eGFP(pEGFPc $\left.c_{1}\right), \mathrm{HA}(\mathrm{pcDNA} 3-\mathrm{HA})$ or His(pET-30a) at the N-terminus of the fusion protein. The amino acid of $\Delta C C$ is 1-600; SH3 1-331; PRC 334-665; PR 334-600; CC 594-665; and SH3C is SH3 plus CC. Plasmids of Alix, together with Alix NT (1-434), Alix CT(468-869), Alix $\Delta$ PRD(1-717), and Alix CT $\Delta$ PRD(468-717), were kindly provided by Dr Re' my Sadoul (Universite' Joseph Fourier, France).

The two CIN85 siRNA sequences were 5'-ACU UUG UAA GAG AAA UAA ATT-3' (labeled as $S 1$ ) and 5'-GGA CAA AGA GCA AGG AUU ATT-3' (labeled as $S 2$ ).
COS-7, HEK293 and 3T3-L1 cells were maintained in DMEM supplemented with $10 \%$ calf serum. Lipofectamine 2000 (Invitrogen) was used for all nucleic acid transfections in accordance with the manufacturer's protocol.

\section{Immunoprecipitation, western blotting and immunofluores- cent staining}

Cells were co-transfected with the two indicated plasmids in the ratio of 1:3 (primary immunoprecipitated protein:co-precipitated protein) and lysed in a buffer containing $1 \%$ Triton X-100, $10 \%$ glycerol, $20 \mathrm{mM}$ Hepes, pH 7.4, $150 \mathrm{mM} \mathrm{NaCl}, 1 \mathrm{mM}$ EDTA, 1 mM EGTA, $1 \mathrm{mM} \mathrm{Na}_{3} \mathrm{VO}_{4}, 1 \mathrm{mM}$ PMSF and $2 \mu \mathrm{l} / \mathrm{ml}$ protease inhibitor cocktails 1 and 2 . The immunoprecipitation and western blots were conducted as described previously with appropriate antibodies [38].

For immunofluorescent staining, cells were fixed in either methanol or $4 \%$ paraformaldehyde (PFA) in phosphate-buffered saline (PBS). eGFP and DsRed fusion proteins or Alexa Fluorlabeled EGF were directly visualized by confocal microscopy. Other proteins were stained with appropriate primary antibodies and fluorescently labeled secondary antibodies.

\section{EGF stimulated EGFR downregulation}

For EGFR endocytosis analysis, COS-7 cells transfected with a plasmid expressing eGFP, eGFP-CIN85 or eGFP- $\triangle \mathrm{CC}$ were serum-starved for $24 \mathrm{~h}$. Cells were stimulated with $25 \mathrm{ng} / \mathrm{ml} \mathrm{EGF}$ for $0,7.5,15$ or $30 \mathrm{~min}$, washed three times with acidic buffer $(150 \mathrm{mM} \mathrm{NaCl}$ and $25 \mathrm{mM}$ acetic acid) and four times with icecold PBS, then lifted by incubation with $2.5 \mathrm{mM}$ EDTA in PBS for 5 min on ice. Fixation was carried out in 4\% PFA in PBS without Triton X-100. After blocking with $1 \%$ bovine serum albumin (BSA) for $1 \mathrm{~h}$, cells were incubated with $10 \mu \mathrm{g} / \mathrm{ml}$ anti-EGFR antibody overnight at $4{ }^{\circ} \mathrm{C}$ and a phycoerythrin (PE)-conjugated secondary antibody for $2 \mathrm{~h}$ at room temperature. Cells were sorted by eGFP and PE double fluorescence. The eGFP-positive cells were analyzed with the Flowjo program for PE fluorescence intensity.

For downregulation analysis of EGFR, serum-starved COS-7 cells that had been stimulated with $25 \mathrm{ng} / \mathrm{ml}$ EGF were harvested in SDS sample buffer and subjected to immunoblotting.

EGF degradation was analyzed following the methods of Harald Stenmark [39] and Phillip Woodman [40] with slight modifications. Briefly, COS-7 cells were transfected with either the CIN85 siRNA $(S 1, S 2)$ or the negative control sequence $(N C) .72 \mathrm{~h}$ posttransfection, the cells that had been serum-starved overnight were incubated with Alexa fluor-labeled EGF $(200 \mathrm{ng} / \mathrm{ml}$ in DMEM supplemented with $20 \mathrm{mM}$ Hepes and $2 \mathrm{mg} / \mathrm{ml} \mathrm{BSA}$ ) on ice for 60 $\mathrm{min}$ and then transferred to a $37{ }^{\circ} \mathrm{C}$ incubator for $20 \min (N C)$ or 60 min (siRNA-transfected cells: S1, S2), at which point the control and siRNA-transfected cells had comparable levels of internalized EGF. Cells were washed with pre-warmed binding buffer $\left(37^{\circ} \mathrm{C}\right)$ three times and released for 30,60 or $180 \mathrm{~min}$. Before fixation with $4 \%$ PFA, cells were permeabilized with $0.05 \%$ saponin (in PBS). The permeabilized fixed cells were analyzed by widefield confocal microscopy. Images were acquired with identical exposure settings, and average EGF fluorescence intensities were calculated for each sight view. These values were normalized to the average value obtained following a release of $30 \mathrm{~min}$.

For EGF pulse-chase analysis, COS-7 cells that had been transfected with GFP-CIN85 or GFP- $\Delta$ CC were incubated with Alexa 
fluor-labeled EGF (in DMEM supplemented with 20 mM HEPES and $2 \mathrm{mg} / \mathrm{ml} \mathrm{BSA}$ ) for $30 \mathrm{~min}$ on ice and then transferred to a 37 ${ }^{\circ} \mathrm{C}$ incubator for the indicated length of time before fixation. Alternatively, the cells were washed with and incubated in $37^{\circ} \mathrm{C}$ binding buffer and subjected to live video microscopy.

\section{PIP strip membrane analysis and liposome association}

eGFP-CIN85 or eGFP- $\triangle$ CC was expressed in HEK293 cells and purified by use of an anti-CIN85 antibody cross-linked to agarose. His-tagged $\mathrm{SH} 3$ domains or PRC were expressed in E. coli and purified by Ni-NTA chromatography. The PIP Strips ${ }^{\mathrm{TM}}$ were used in accordance with the manufacturer's recommendations.

For liposome association analysis, $300 \mu \mathrm{g}$ phosphatidyl choline or $270 \mu \mathrm{g}$ phosphatidyl choline plus $30 \mu \mathrm{g}$ phosphatidic acid was dissolved in $300 \mu \mathrm{l}$ chloroform and then dried by nitrogengas flow. Liposomes were prepared by resuspending the lipids in $300 \mu \mathrm{l}$ buffer $(0.25 \mathrm{M}$ sucrose, $20 \mathrm{mM}$ Hepes, $\mathrm{pH} 7.4,0.1 \%$ $\beta$-mercaptoethanol, $1 \mathrm{mM} \mathrm{PMSF}$ and $2 \mu \mathrm{l} / \mathrm{ml}$ protease inhibitor cocktails 1 and 2) and sonicating to clearance. For each trial, a similar mole (about $0.2 \mathrm{nmol}$ ) of purified protein was added to the indicated liposome solution and the solution was incubated at $4{ }^{\circ} \mathrm{C}$ overnight. The liposome-protein solution was ultracentrifuged for $1 \mathrm{~h}$ at $200000 \mathrm{~g}$ and the resultant supernatant and pellet were collected for western blotting.

\section{Cell fractionation with discontinuous sucrose density gradient centrifugation}

Cells were homogenized in isotonic buffer containing $20 \mathrm{mM}$ Hepes, pH 7.4, 0.25 M sucrose, $1 \mathrm{mM}$ EDTA, $2.5 \mathrm{mM}$ dithiothreitol, $1 \mathrm{mM}$ PMSF and $2 \mu \mathrm{l} / \mathrm{ml}$ protease inhibitor cocktails 1 and 2. Each total cell lysate was fractionated by using a discontinuous sucrose gradient composed of $1 \mathrm{ml} 0.6 \mathrm{M}$ sucrose, $1 \mathrm{ml} 0.86 \mathrm{M}$ sucrose, $1.5 \mathrm{ml} 1.06 \mathrm{M}$ sucrose, $1.5 \mathrm{ml} 1.18 \mathrm{M}$ sucrose, $2.5 \mathrm{ml} 1.4$ $\mathrm{M}$ sucrose and $1.5 \mathrm{ml} 2 \mathrm{M}$ sucrose, and then centrifuged at 100 $000 \mathrm{~g}$ for $18 \mathrm{~h}$. For fractionation of the cytoplasm and intracellular vesicle membranes, each cell homogenate was centrifuged at $12000 \mathrm{~g}$ for $15 \mathrm{~min}$. The pelleted cell debris and large membrane pieces were discarded. The supernatant was then ultracentrifuged at $200000 \mathrm{~g}$ for $90 \mathrm{~min}$ to separate the cytoplasm and intracellular vesicle membranes.

\section{Acknowledgments}

We thank Dr Margo Myers (UCSF, USA) for providing the plasmid of c-Cbl, Dr Re'my Sadoul (Universite' Joseph Fourier, France) for providing the plasmids of Alix and truncated forms of Alix, and Dr Uta von Schwedler (University of Utah, USA) for providing the plasmids of Vps4 and Vps4 M, and CHMP4B. This work was supported by grants 90208007,30521005 and 30623002 from the National Nature Sciences Foundation of China, and grants 2002CB513000 and 2006CB910700 from the Ministry of Sciences and Technology of China.

\section{References}

1 Dikic I. CIN85/CMS family of adaptor molecules. FEBS Lett 2002; 529:110-115.

2 Take H, Watanabe S, Takeda K, Yu ZX, Iwata N, Kajigaya
S. Cloning and characterization of a novel adaptor protein, CIN85, that interacts with c-Cbl. Biochem Biophys Res Commun 2000; 268:321-328.

3 Thien CB, Langdon WY. Cbl: many adaptations to regulate protein tyrosine kinases. Nat Rev Mol Cell Biol 2001; 2:294307.

4 Soubeyran P, Kowanetz K, Szymkiewicz I, Langdon WY, Dikic I. Cbl-CIN85-endophilin complex mediates ligand-induced downregulation of EGF receptors. Nature 2002; 416:183-187.

5 Petrelli A, Gilestro GF, Lanzardo S, Comoglio PM, Migone $\mathrm{N}$, Giordano S. The endophilin-CIN85-Cbl complex mediates ligand-dependent downregulation of c-Met. Nature 2002; 416:187-190.

6 Di Fiore PP, De Camilli P. Endocytosis and signaling. An inseparable partnership. Cell 2001; 106:1-4.

7 Haglund K, Shimokawa N, Szymkiewicz I, Dikic I. Cbl-directed monoubiquitination of CIN85 is involved in regulation of ligand-induced degradation of EGF receptors. Proc Natl Acad Sci USA 2002; 99:12191-12196.

8 Katzmann DJ, Odorizzi G, Emr SD. Receptor downregulation and multivesicular-body sorting. Nat Rev Mol Cell Biol 2002; 3:893-905.

9 Raiborg C, Rusten TE, Stenmark H. Protein sorting into multivesicular endosomes. Curr Opin Cell Biol 2003; 15:446-455.

10 Kowanetz K, Szymkiewicz I, Haglund K, et al. Identification of a novel proline-arginine motif involved in CIN85-dependent clustering of $\mathrm{Cbl}$ and down-regulation of epidermal growth factor receptors. J Biol Chem 2003; 278:39735-39746.

11 Simpson F, Hussain NK, Qualmann B, et al. SH3-domaincontaining proteins function at distinct steps in clathrin-coated vesicle formation. Nat Cell Biol 1999; 1:119-124.

12 Huttner WB, Schmidt A. Lipids, lipid modification and lipidprotein interaction in membrane budding and fission--insights from the roles of endophilin A1 and synaptophysin in synaptic vesicle endocytosis. Curr Opin Neurobiol 2000; 10:543-551.

13 Burkhard P, Stetefeld J, Strelkov SV. Coiled coils: a highly versatile protein folding motif. Trends Cell Biol 2001; 11:8288.

14 Kirsch KH, Georgescu MM, Ishimaru S, Hanafusa H. CMS: an adapter molecule involved in cytoskeletal rearrangements. Proc Natl Acad Sci USA 1999; 96:6211-6216.

15 Gaidos G, Soni S, Oswald DJ, Toselli PA, Kirsch KH. Structure and function analysis of the CMS/CIN85 protein family identifies actin-bundling properties and heterotypic-complex formation. J Cell Sci 2007; 120 (Pt 14):2366-2377.

16 Kowanetz K, Husnjak K, Holler D, et al. CIN85 associates with multiple effectors controlling intracellular trafficking of epidermal growth factor receptors. Mol Biol Cell 2004; 15:3155-3166.

17 Szymkiewicz I, Shupliakov O, Dikic I. Cargo- and compartment-selective endocytic scaffold proteins. Biochem J 2004; 383:1-11.

18 Whitley P, Reaves BJ, Hashimoto M, Riley AM, Potter BV, Holman GD. Identification of mammalian Vps24p as an effector of phosphatidylinositol 3,5-bisphosphate-dependent endosome compartmentalization. J Biol Chem 2003; 278:3878638795.

19 Lin Y, Kimpler LA, Naismith TV, Lauer JM, Hanson PI. Interaction of the mammalian endosomal sorting complex 
required for transport (ESCRT) III protein hSnf7-1 with itself, membranes, and the AAA+ ATPase SKD1. J Biol Chem 2005; 280:12799-12809.

20 Jozic D, Cardenes N, Deribe YL, et al. Cbl promotes clustering of endocytic adaptor proteins. Nat Struct Mol Biol 2005; 12:972-979.

21 Möbius W, van Donselaar E, Ohno-Iwashita Y, et al. Recycling compartments and the internal vesicles of multivesicular bodies harbor most of the cholesterol found in the endocytic pathway. Traffic 2003; 4:222-231

22 Szymkiewicz I, Kowanetz K, Soubeyran P, Dinarina A, Lipkowitz S, Dikic I. CIN85 participates in Cbl-b-mediated downregulation of receptor tyrosine kinases. J Biol Chem 2002; 277:39666-39672.

23 Morino C, Kato M, Yamamoto A, et al. A role for Hrs in endosomal sorting of ligand-stimulated and unstimulated epidermal growth factor receptor. Exp Cell Res 2004; 297:380-391.

24 Williams RL, Urbe S. The emerging shape of the ESCRT machinery. Nat Rev Mol Cell Biol 2007; 8:355-368.

25 Yoshimori T, Yamagata F, Yamamoto A, et al. The mouse $\mathrm{SKD} 1$, a homologue of yeast Vps4p, is required for normal endosomal trafficking and morphology in mammalian cells. Mol Biol Cell 2000; 11:747-763.

26 Fujita H, Yamanaka M, Imamura K, et al. A dominant negative form of the AAA ATPase SKD1/VPS4 impairs membrane trafficking out of endosomal/lysosomal compartments: class E vps phenotype in mammalian cells. J Cell Sci 2003; 116 (Pt 2):401-414.

27 Liscovitch M. Phospholipase D: role in signal transduction and membrane traffic. J Lipid Mediat Cell Signal 1996; 14:215221.

28 Jenkins GM, Frohman MA. Phospholipase D: a lipid centric review. Cell Mol Life Sci 2005; 62:2305-2316.

29 Stace CL, Ktistakis NT. Phosphatidic acid- and phosphatidylserine-binding proteins. Biochim Biophys Acta 2006; 1761:913-926.
30 Shen Y, Xu L, Foster DA. Role for phospholipase D in receptor-mediated endocytosis. Mol Cell Biol 2001; 21:595-602.

31 Zhao C, Du G, Skowronek K, Frohman MA, Bar-Sagi D. Phospholipase D2-generated phosphatidic acid couples EGFR stimulation to Ras activation by Sos. Nat Cell Biol 2007; 9:706-712.

32 Hughes WE, Parker PJ. Endosomal localization of phospholipase $\mathrm{D} 1 \mathrm{a}$ and $1 \mathrm{~b}$ is defined by the $\mathrm{C}$-termini of the proteins, and is independent of activity. Biochem $J$ 2001; 356:727-736.

33 McDermott M, Wakelam MJ, Morris AJ. Phospholipase D. Biochem Cell Biol 2004; 82:225-253.

34 Cormont M, Meton I, Mari M, et al. CD2AP/CMS regulates endosome morphology and traffic to the degradative pathway through its interaction with Rab4 and c-Cbl. Traffic 2003; 4:97-112.

35 Welsch T, Endlich N, Gokce G, et al. Association of CD2AP with dynamic actin on vesicles in podocytes. Am J Physiol Renal Physiol 2005; 289:F1134-F1143.

36 Matsuo H, Chevallier J, Mayran N, et al. Role of LBPA and Alix in multivesicular liposome formation and endosome organization. Science 2004; 303:531-534.

37 Schmidt MH, Hoeller D, Yu J, et al. Alix/AIP1 antagonizes epidermal growth factor receptor downregulation by the CblSETA/CIN85 complex. Mol Cell Biol 2004; 24:8981-8993.

38 Huo H, Guo X, Hong S, Jiang M, Liu X, Liao K. Lipid rafts/ caveolae are essential for insulin-like growth factor-1 receptor signaling during 3T3-L1 preadipocyte differentiation induction. J Biol Chem 2003; 278:11561-11569.

39 Doyotte A, Mironov A, McKenzie E, Woodman P. The Bro1related protein HD-PTP/PTPN23 is required for endosomal cargo sorting and multivesicular body morphogenesis. Proc Natl Acad Sci USA 2008; 105:6308-6313.

40 Raiborg C, Bache KG, Mehlum A, Stang E, Stenmark H. Hrs recruits clathrin to early endosomes. EMBO J 2001; 20:50085021 .

(Supplementary information is linked to the online version of the paper on the Cell Research website.) 